\title{
Arterio-colonic Fistula as a Complication of Failed Pancreatic Transplant
}

\author{
Mantilla $\mathrm{N}^{1,2^{*}}$, Calata $\mathrm{J}^{1,2}$, Trepanier $\mathrm{JS}^{1,2}$, Tzvetanov $\mathrm{I}^{3}$, Nordenstam $\mathrm{J}^{1}$, Chaudhry $\mathrm{V}^{1,2}$ and Mellgren $\mathrm{A}^{1}$ \\ ${ }^{1}$ Department of Surgery, Division of Colon \& Rectal Surgery, University of Illinois at Chicago, USA \\ ${ }^{2}$ Colorectal Surgery Department, John H Stroger, Jr Hospital of Cook County, USA \\ ${ }^{3}$ Department of Surgery, Division of Transplant Surgery, University of Illinois at Chicago, USA

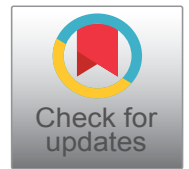

*Corresponding author: Nathalie Mantilla, MD, Department of Surgery, Division of Colon \& Rectal Surgery, University of Illinois at Chicago, USA; Colorectal Surgery Fellow, Colorectal Surgery Department, John H. Stroger, Jr. Hospital of Cook County, 1900 West Polk Street, G-23 Admin Building, Room 401, Chicago, IL, USA, E-mail: nathaliemantilla@gmail.com

\begin{abstract}
Arterio-enteric fistulas are rare but feared complications after failed enterically drained pancreatic transplants. Massive and abrupt gastrointestinal bleeding is the cardinal presentation as a result of pseudoaneurysm rupture into the bowel lumen. However, subclinical presentation of delayed arterio-enteric fistulas may represent a diagnostic challenge for surgeons and warrants a high index of suspicion. Endovascular techniques have evolved as an excellent option as a bridge to definitive treatment.
\end{abstract}

\section{Keywords}

Arterio-enteric fistula, Failed pancreatic transplant, Gastrointestinal bleeding, Endovascular stenting

\section{Introduction}

Arterio-enteric fistulas are rare but feared complications after failed enterically drained pancreatic transplants. Although massive gastrointestinal bleeding is the cardinal presentation after pseudoaneurysm rupture into the bowel lumen, not all patients present similarly. Organ wall erosion by leaked pancreatic enzymes is one of the key pathophysiologic elements of this condition. We present a case of a subclinical manifestation of an iliac artery to sigmoid colon fistula after a failed pancreas transplantation. The delayed nature and slow progression of this arterio-colonic fistula made the diagnosis and management particularly challenging.

\section{Case}

Our patient is a 49-year-old male with a long history of insulin-dependent diabetes mellitus (IDDM) complicated by diabetic nephropathy and end-stage renal disease (ESRD) on hemodialysis. After a standard preoperative pre-transplant process, he was found to have a matched cadaveric donor and underwent a combined kidney and pancreas transplant. The pancreatic graft was enterically drained and the kidney was placed in the left lower abdomen in the traditional manner. The first two weeks of postoperative course were complicated by pancreatic graft thrombosis and ureteral leak, requiring pancreatectomy, right common iliac artery repair with a bovine patch, and repair of the ureteral leak with nephrostomy tube placement. After being discharged from the hospital, he had a slow recovery, requiring close monitoring and frequent follow up appointments. Fourteen months after the initial surgery, he presented to the emergency room complaining of abdominal pain after a fall. Due to concern of organ damage from blunt trauma to the abdomen, a computerized tomography scan (CT) of the abdomen and pelvis with intravenous contrast was performed which showed a pseudoaneurysm arising off the right common iliac artery with free pelvic fluid concerning for an associated leak (Figure 1). There was also demarcation of the sigmoid colon with contrast despite the lack of administration of oral contrast. No definitive fistulous tract was seen; however, an arterio-enteric fistula was highly suspected based on the history of the arterial wall patch repair. The patient remained hemodynamically stable without evidence of gastrointestinal bleeding. Vascular surgery was consult-

Citation: Mantilla N, Calata J, Trepanier JS, Tzvetanov I, Nordenstam J, et al. (2018) Arterio-colonic Fistula as a Complication of Failed Pancreatic Transplant. Int J Surg Res Pract 5:069. doi.org/10.23937/23783397/1410069

Received: April 07, 2018: Accepted: May 17, 2018; Published: May 19, 2018

Copyright: (C) 2018 Mantilla N, et al. This is an open-access article distributed under the terms of the Creative Commons Attribution License, which permits unrestricted use, distribution, and reproduction in any medium, provided the original author and source are credited. 
ed, and an emergency retrograde pelvic angiogram and aortogram was performed through an ipsilateral catheterization of right external iliac artery. Identification of a pseudoaneurysm adjacent to the repair of the right common iliac artery raised concern of a pressure-erosion mechanism for the creation of the arterio-colonic fistula, which at the time was not involving all the layers of the bowel wall, but instead dissecting through the sigmoid colon wall. Because of these particular findings of the fistula, with partial colonic wall involvement, the decision was made to place an intravascular stent graft

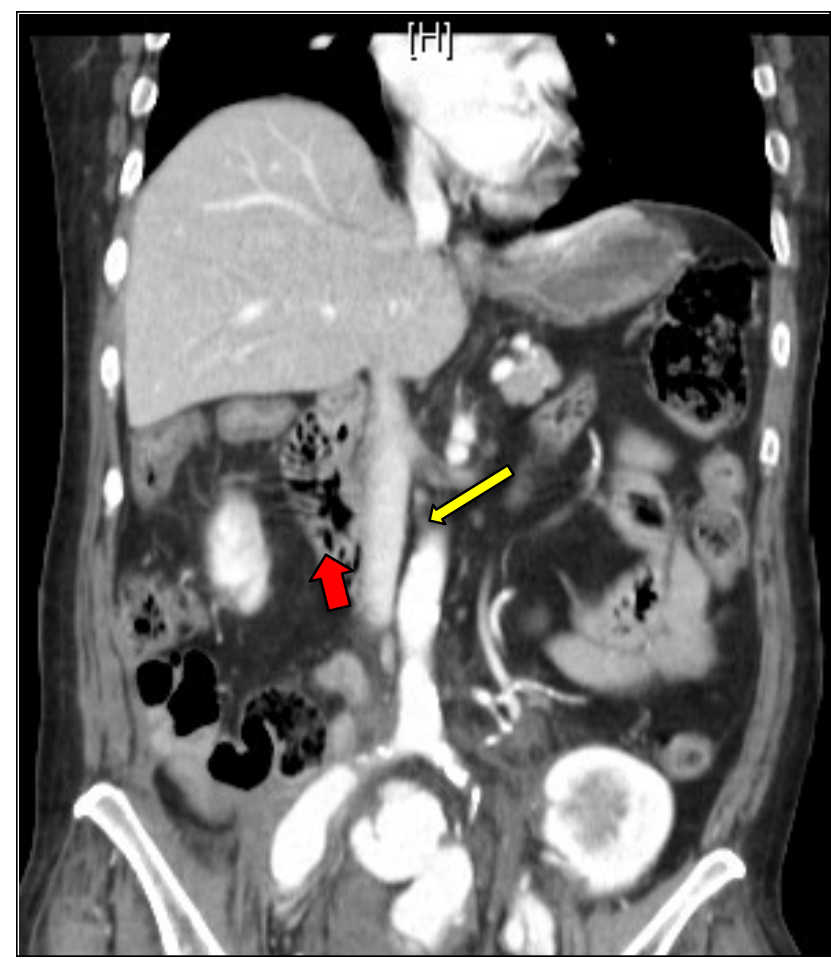

Figure 1: Computer tomography scan of abdomen and pelvis with intravenous contrast, coronal view. Demonstrating a pseudoaneurysm arising off right common iliac artery (short red arrow) with free pelvic fluid concerning for an associated leak from the pseudoaneurysm. Demarcation of the sigmoid colon with contrast (long yellow arrow) in close proximity to the pseudoaneurysm. to cover the area of connection between the pseudoaneurysm and the sigmoid colon. An exclusion stent graft was deployed in the right common iliac/external iliac artery pseudoaneurysm, utilizing the same system used for the endovascular treatment of infrarenal abdominal aortic or aortoiliac aneurysms (Endurant $\|^{\circledR}$ contralateral limb stent graft; $16 \times 10 \times 156 \mathrm{~mm}$ - Medtronic) as shown in Figure 2. This procedure was performed as a temporary control measure, to decrease the possibilities of a massive lower gastrointestinal bleeding. Although a true communication with the colonic mucosa and bowel lumen was not proven, the likelihood of graft infection was assumed to be high, therefore, once having a better control of the fistula, the plan was to proceed with definitive surgical repair in the near future.

Colorectal surgery was consulted, and a bedside flexible sigmoidoscopy performed. A sub-mucosal hematoma was found on a $10-\mathrm{cm}$ segment of the sigmoid ap-

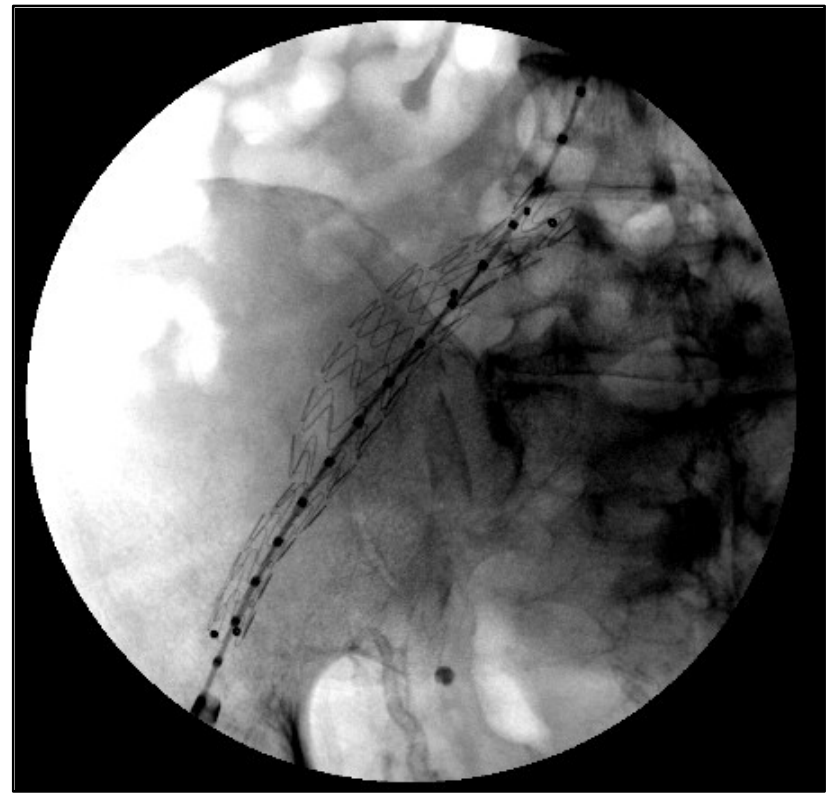

Figure 2: Placement of an exclusion stent graft in the right common and external iliac arteries.
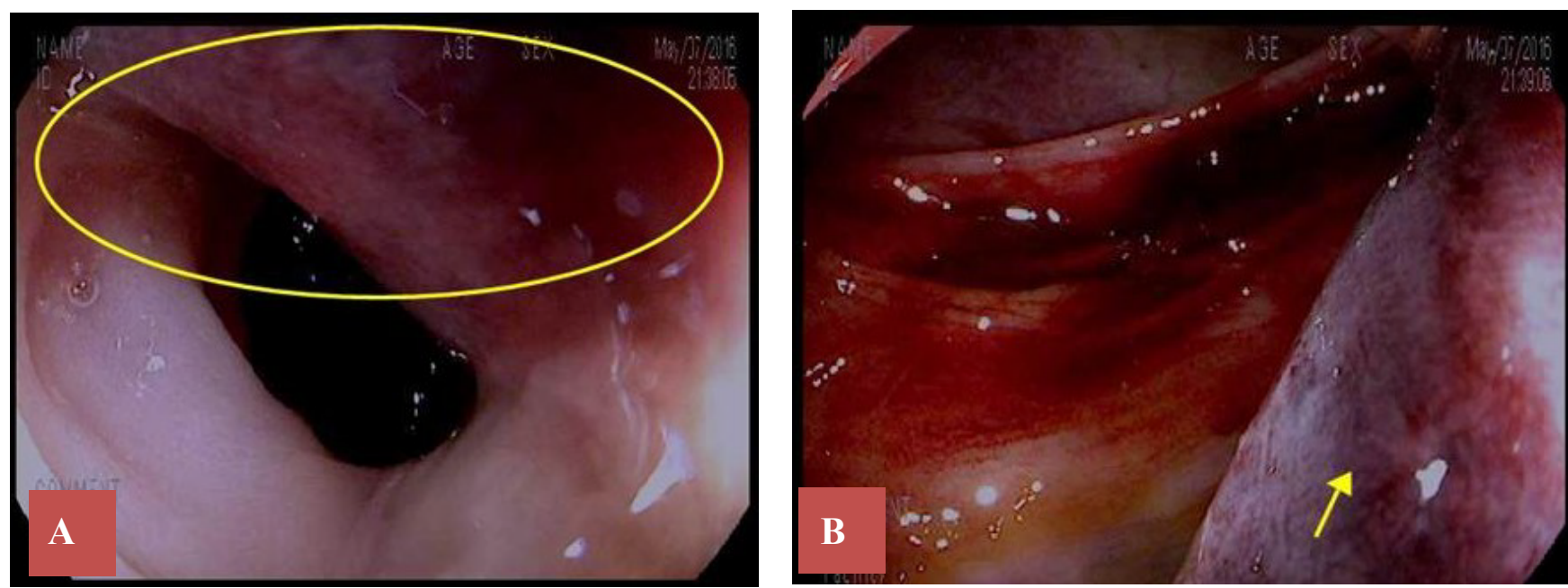

Figure 3: A) Area of suspected fistula encircled; B) Sub-mucosal hematoma between $25-35 \mathrm{~cm}$ from anal verge (arrow). 
proximately $25-35 \mathrm{~cm}$ from the anal verge. No intraluminal bleeding was seen, but a fresh adherent clot was identified in the suspected fistula region (Figure $3 \mathrm{~A}$ and Figure 3B).

The patient remained stable and was discharged after clinical parameters were met. One month later, a flexible sigmoidoscopy was performed showing nearly complete resolution of the sub-mucosal hematoma without evidence of intraluminal blood (Figure 4A and Figure 4B). Additionally, a pelvic CT angiogram with 3-dimensional reconstruction confirmed stable location of the vascular stent graft without presence of contrast in the colonic wall (Figure 5A and Figure 5B). He was subsequently scheduled for a partial colectomy and vascular reconstruction of the right iliac artery.

\section{Discussion}

About 947 pancreatic transplantations were performed in 2015 in the United States, and nearly 75\% are associated with kidney transplantation [1]. Over the last few decades, there has been a significant decrease in complications after pancreatic transplant; however, pancreatic transplantation still carries an overall high morbidity. More than $70 \%$ of early pancreatic graft losses are related to technical failures [2], with graft thrombosis as the most common cause $(3-10 \%)$ such as in the case we report. Other complications can be encountered depending on the drainage technique [3]. Common complications in bladder-drained cases include recurrent urinary tract infections, urethritis, metabolic alkalosis, calculi, cystitis and hematuria. Enteric drainage of the exocrine pancreas is an excellent alternative option, given the low rate of complications compared with the bladder-drainage method. Unfortunately, if complications occur with this technique, they can result in a fatal situation. Arterio-enteric fistulas are rare but feared complications after failed enteric-drained pancreatic
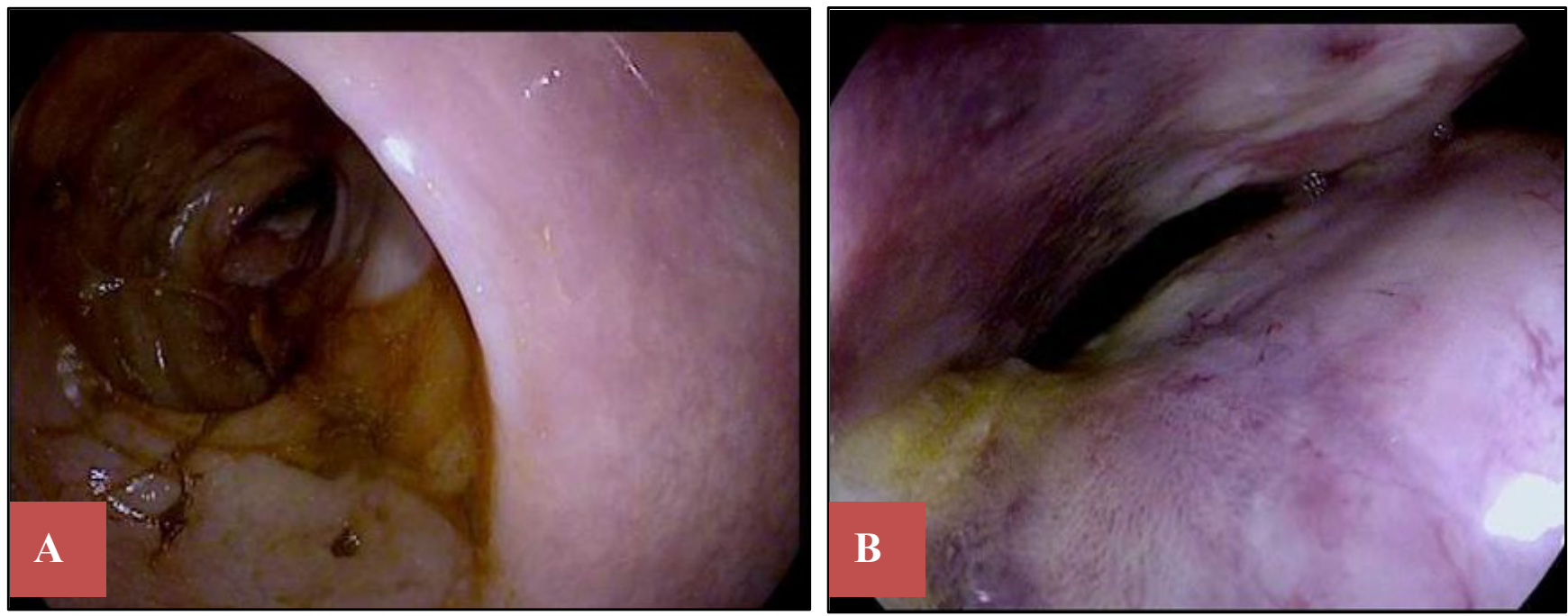

Figure 4: Flexible sigmoidoscopy (one month after endovascular stenting), showing almost complete resolution of the sub-mucosal hematoma without evidence of intraluminal blood.
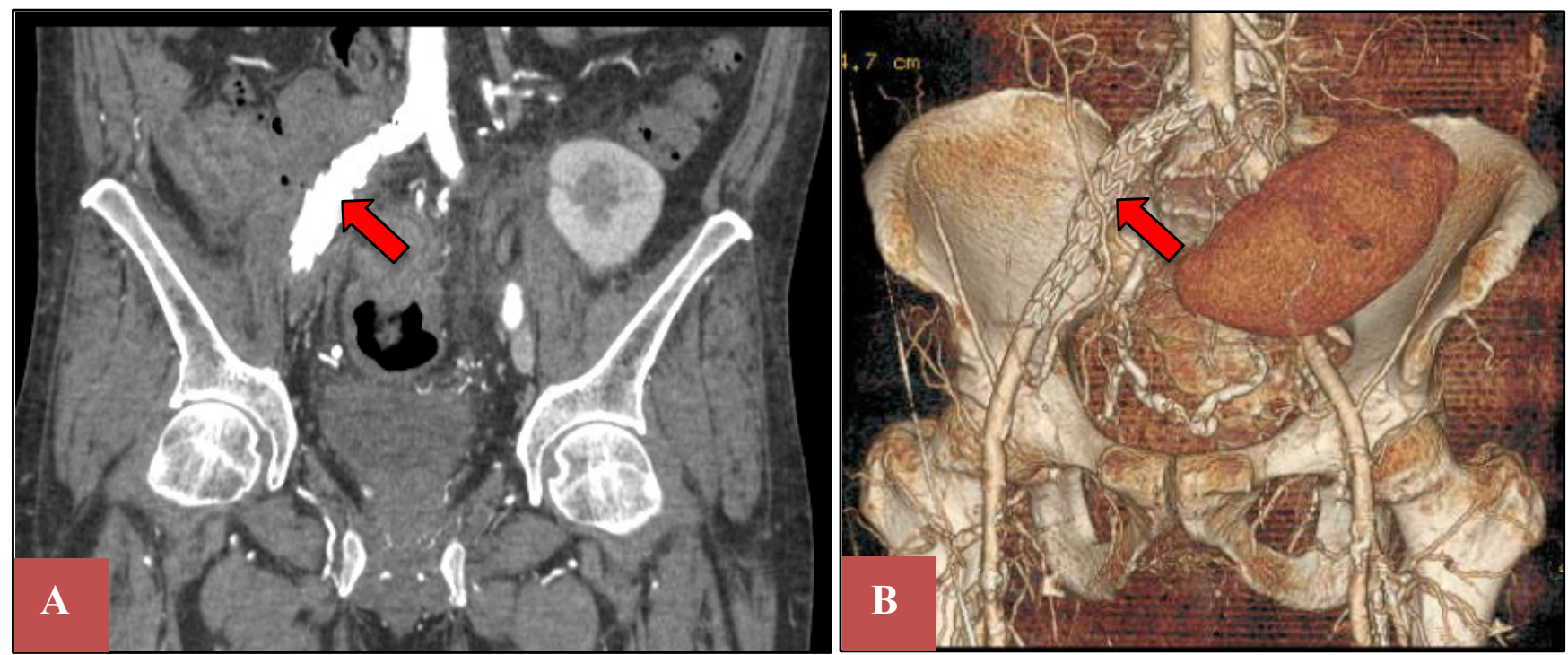

Figure 5: Pelvic CT angiogram with 3-dimensional reconstruction showing in-situ vascular stent graft (red arrows) without extravasation of contrast in the colonic wall. 
transplantation. Due to its infrequency, there is no clear knowledge of its incidence, and most of the understanding of this condition is based on case reports and single institution experiences. Chemical erosion of a vessel wall from leakage of digestive pancreatic enzymes after a failed pancreatic enteric-drained pancreatic graft may result in an arterio-enteric fistula. In our case, we speculate it was due to a combination of factors, prompted by enzymatic erosion of a repaired arterial wall, facilitating the creation of a pseudoaneurysm. Furthermore, the proximity of the colonic wall, and the pulsatile pressure over it may have aided the creation of the arterio-colonic fistula. This type of complication typically presents as massive and abrupt gastrointestinal bleeding from pseudoaneurysm rupture $[4,5]$. In our case, the subtle presentation with an absence of gastrointestinal bleeding easily could have led to a different, fatal outcome. Prompt recognition of the fistula on axial imaging as well as immediate endovascular intervention were crucial in the successful management of this patient. In our case, we cannot pinpoint the exact time of fistula development in relation to his last surgery. The presence of abdominal pain may have been only related to trauma after his fall, but undoubtedly it was an essential part of the diagnostic process in the absence of other clinical findings. Therefore, a high index of suspicion and prompt work up including endoscopic evaluation and imaging studies are warranted. In cases of massive bleeding, resuscitation remains the essential initial step. A recent review of the literature by Villa, et al. found 11 cases of iliac-enteric fistulas in failed enteric-drained pancreas transplant [5]. Sixty-four percent of the patients presented with upper gastrointestinal bleed, and mortality was $27 \%$. Seven patients were initially managed with endovascular techniques ( 5 stents, 2 embolization's).

Successful endovascular intervention in these types of cases has been reported in various publications [610]. This modality offers a bridge to definitive treatment by allowing for temporary control of bleeding while giving time to medically optimize the patient and delineate the anatomy before surgery. In our case, an endovascular approach allowed us to primarily prevent a devastating bleeding, while planning for definitive surgery. Active brisk bleeding and hemodynamic instability are indicators of poor prognosis and may mandate an urgent surgical intervention. Given the complexity and severity of these cases, an early multispecialty approach is advised, including transplant surgeons, general or colorectal surgeons, interventional radiologists and vascular surgeons.

\section{References}

1. 2015 Annual Data Report. Scientific Registry of Transplant Recipients.

2. Troppmann C (2010) Complications after pancreas transplantation. Curr Opin Organ Transplant 15: 112-118.

3. Demartines N, Schiesser M, Clavien PA (2005) An evidence-based analysis of simultaneous pancreas-kidney and pancreas transplantation alone. Am J Transplant 5: 2688-2697.

4. Barone GW, Webb JW, Hudec WA (1998) The enteric drained pancreas transplant: another potential source of gastrointestinal bleeding. Am J Gastroenterol Aug 93: 1369-1371.

5. Villa M, Siskind E, Jaimes N, Eckstein D, Bhaskaran M, et al. (2014) Arterio-Enteric Fistula in Failed Enteric-Drained Pancreas Transplants: An Impending Danger. Int J Angiol 23: 65-68.

6. Dalla Valle R, Capocasale E, Mazzoni MP, Busi N, Piazza $P$, et al. (2005) Embolization of a ruptured pseudoaneurysm with massive hemorrhage following pancreas transplantation: a case report. Transplant Proc 37: 2275-2277.

7. Verni MP, Leone JP, DeRoover A (2001) Pseudoaneurysm of the Y-graft/iliac artery anastomosis following pancreas transplantation: a case report and review of the literature. Clin Transplant 15: 72-76.

8. Harle P, Schwarz S, Langgartner J, Scholmerich J, Rogler G (2010) Volcano-like intermittent bleeding activity for seven years from an arterio-enteric fistula on a kidney graft site after pancreas-kidney transplantation: a case report. J Med Case Reports 4: 357-359.

9. Paduch DA, Conlin M, Dematos A, Rabkin J, Orloff S, et al. (2000) Arterial duodenovesical fistula after simultaneous pancreas and kidney transplantation. J Urol 164: 1296.

10. Orsenigo E, De Cobelli F, Salvioni M, Cristallo M, Fiorina P, et al. (2003) Successful endovascular treatment for gastroduodenal artery pseudoaneurysm with an arteriovenous fistula after pancreas transplantation. Transpl Int 16: 694-696. 